\title{
News and Reports: Identification of the rs797045105 in the SERAC1 Gene by Whole-exome Sequencing in a Patient Suspicious of MEGDEL Syndrome
} \author{
aghat $^{1,3^{*}} \oplus$, Gholamreza Shariati ${ }^{1,4^{*}} \oplus$, Hamid Galehdari ${ }^{1,2}$ (i) \\ 1. Narges Medical Genetics and Prenatal Diagnosis Laboratory, Ahvaz, Iran. \\ 2. Department of Genetics, Faculty of Science, Shahid Chamran University of Ahvaz, Ahvaz, Iran. \\ 3. Diabetes Research Center, Ahvaz Jundishapur University of Medical Sciences, Ahvaz, Iran. \\ 4. Department of Genetics, School of Medicine, Ahvaz Jundishapur University of medical Sciences, Ahvaz, Iran.
}

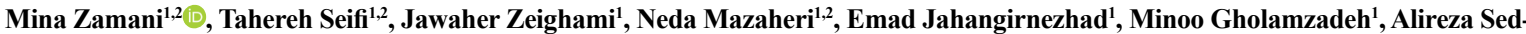

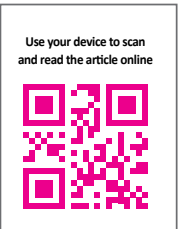

ditation: Zamani, M., Seifi, T., Zeighami, J., Mazaheri, J., Mazaheri, N., \& Jahangirnezhad, F., et al. (2020). Identification of the rs797045105 in the SERAC1 Gene by Whole-exome Sequencing in a Patient Suspicious of MEGDEL Syndrome. Basic and Clinical Neuroscience, 11(4), 549-556. http://dx.doi.org/10.32598/bcn.9.10.455

doi) http://dx.doi.org/10.32598/ben.9.10.455

\section{(1) (3)}

Article info:

Received: 13 Oct 2017

First Revision: 10 Nov 2017

Accepted: 13 May 2019

Available Online: $01 \mathrm{Jul} 2020$

Keywords:

Whole-exome sequencing, rs797045105, SERAC1, MEGDEL

\section{A B S T RA C T}

Introduction: Whole Exome Sequencing (WES) has been increasingly utilized in genetic determinants of various inherited diseases.

Methods: We applied WES for a patient presenting 3-Methylglutaconic Aciduria (MEG), Deafness (D), Encephalopathy (E), and Leigh-like (L) syndrome. Then Sanger sequencing was used for the detected variant validation.

Results: We found an insertion, rs797045105 (chr6, 158571484, C>CCATG), in the SERAC1 gene with homozygous genotype in the patient and heterozygous genotype in her unaffected parents. Notably, bioinformatics analysis using mutation taster (prob $>0.99$ ) and DDIGin (prob=86.51) predicted this mutation as disease-causing. Also, the variant was not present in our database, including 700 exome files.

Conclusion: These findings emphasize the pathogenicity of rs797045105 for MEGDEL syndrome. On the other hand, our data shed light on the significance of WES application as a genetic test to identify and characterize the comprehensive spectrum of genetic variation and classification for patients with neuro- metabolic disorders.

\footnotetext{
* Corresponding Author:

Hamid Galehdari, PhD.

Address: Department of Genetics, Faculty of Science, Shahid Chamran

University of Ahvaz, Ahvaz, Iran.

Tel: +98 (916) 6149028

E-mail:galehdari187@yahoo.com
}

Gholamreza Shariati, PhD.

Address: : Department of Genetics, Faculty of Science, Shahid Chamran University of Ahvaz, Ahvaz, Iran.

Tel: +98 (912) 1115529

E-mail: gshariati@yahoo.com 


\section{Highlights}

- WES is an applicable tool to evaluate genetically metabolic diseases.

- rs797045105 highly potentially causes MEGDLE syndrome.

- rs797045105 makes a frameshift change in SERAC1 gene.

\section{Plain Language Summary}

The genetic architecture of metabolic traits and diseases is complex. Many genes have role in the pathogenicity of these diseases. Advanced technologies including Whole Exome Sequencing (WES) which can assess many genes simultaneously could be an effective option to find out the mutations causing heterogeneous metabolic disorders. In this study we applied WES for a patient presenting MEGDLE syndrome: a neuro-metabolic disease. After data analysis we found rs 797045105 as a disease causing genetic variant for the patient. This pathogenic variation should be considered in genetic profiling of patients with MEGDLE syndrome especially from Southwest of Iran.

\section{Introduction}

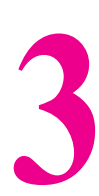

-methylglutaconic aciduria (3-MGA-uria) is a heterogeneous group of metabolic syndromes featured by elevated urinary excretion of 3-methylglutaconic and 3-methyl glutaric acids (Wortmann et al., 2012).

The MEGDEL syndrome (3-MGA-uria type IV) has been introduced by clinical presentations, including neurological deterioration, central nervous system involvement, sensory-neural hearing loss, encephalopathy, and dystonia (Phenotype MIM number: 614739). MEGDEL syndrome is a recessive disorder of dystonia and deafness with Leigh-like syndrome (Wortmann et al., 2006).

MEGDEL syndrome is caused by mutations in serine active site containing 1 (SERAC1). Studies by Wortmann et al. on fibroblasts derived from patients showed that SE$\mathrm{RACl}$ is localized at the interface between mitochondria and endoplasmic reticulum. They showed that mutations in SERAC1 can abnormally increase the ratio of phosphatidylglycerol-34:1 to phosphatidylglycerol-36:1. Therefore, it is speculated that this leads to lower bis (mono-acyl-glycerol) phosphate levels and the accumulation of cholesterol in the perinuclear region. Finally, it is hypothesized that SERAC1 can catalyze the first enzymatic reaction in the bisphosphate(mono-acyl-glycerol) biosynthetic pathway and has a role in the phosphatidylglycerol remodeling pathways as it is involved in the transacylation-acylation reaction to produce phosphatidylglycerol-36:1. They concluded that SERAC1 encoded protein participates in both mitochondrial function and intracellular cholesterol trafficking (Wortmann et al., 2012).
In recent years, Next-Generation Sequencing (NGS) has improved our knowledge about the genetic and molecular bases of this heterogeneous group of disorders.

Here, we used Whole-Exome Sequencing (WES) for genetic diagnosis of an Iranian patient with 3-MGA-uria, mental retardation, cerebellar atrophy, deafness, basal ganglia impairment, and spasticity symptoms, which are compatible with MEGDEL syndrome.

\section{Materials and Methods}

\subsection{Patient/ case report}

A patient with MEGDEL and his parents were recruited from the southwest of Iran.

\subsection{Peripheral blood samples}

For blood sampling, $10 \mathrm{ml}$ of peripheral blood of the patient and his parents were collected in EDTA tubes.

\subsection{DNA extraction}

Genomic DNA was extracted using the standard salting-out protocol. The quality and quantity of the extracted DNA samples were checked by the gel electrophoresis and NanoDrop.

\subsection{Exome sequencing}

The sample was subjected to WES using the IlluminaHiSeq 2000 Genome Analyzer Platform (CNAG) by the Macrogen Company. 
Table 1. Sequences of the used primers

\begin{tabular}{cc}
\hline Name & Sequence \\
\hline SERAC1-F & TGTCCTTGAACAAAAAGTCAGTATGT \\
SERAC1-R & CCTCAATTAATTCTTCAGGAACTTGG \\
SERAC1-RTprimer-F & AAGGCTGTGACATTAGATACTC \\
SERAC1-RTprimer-R1 & AGCAAATGGATTCCGCAGTATC \\
SERAC1-RTprimer-R2 & TGACTTGCTCTTCCGTAGG \\
\hline
\end{tabular}

NEUR SCIENCE

\subsection{RNA extraction, Reverse transcription}

Total RNA was extracted using TRIzol ${ }^{\circledR}$ reagent (Life Technologies). Also, the complementary DNA was synthesized by the PrimeScript ${ }^{\mathrm{TM}}$ RT reagent kit (Takara Bio Inc, Shiga, Japan).

\subsection{Polymerase Chain Reaction (PCR)}

The targeted region of the SERAC1 gene on DNA and cDNA was amplified by PCR using primers designed by Oligo 7 software (Table 1). The PCR was conducted using the Master Mix (Ampliqon, Denmark). The PCR thermal program was as follows: $95^{\circ} \mathrm{C}$ for $5 \mathrm{~min}, 35 \mathrm{cy}$ cles of $95^{\circ} \mathrm{C}$ for $30 \mathrm{~s}, 60^{\circ} \mathrm{C}$ for $30 \mathrm{~s}$, and $72^{\circ} \mathrm{C}$ for $30 \mathrm{~s}$, and $72^{\circ} \mathrm{C}$ for $5 \mathrm{~min}$.

\subsection{DNA Sanger-sequencing}

Ampliqons were directly sequenced by the $\mathrm{ABI}$ PRISM 3700 Genetic Analyzer (Applied Biosystems). The sequences were analyzed with Chromas LITE 2.1.1 and then compared with the reference genomic sequence using BLASTN. Also, the presence of the detected mutation was confirmed by the parent DNA analysis and bi-directional sequencing.

\subsection{In-silico analysis}

Various tools are available for the prediction of pathogenicity of variations in coding and non-coding regions. In the present study, we used some of these tools for the evaluation of disease-causing potentiality of the present insertion variation. The prediction of variant pathogenicity was done using Mutation taster and DDIGin.

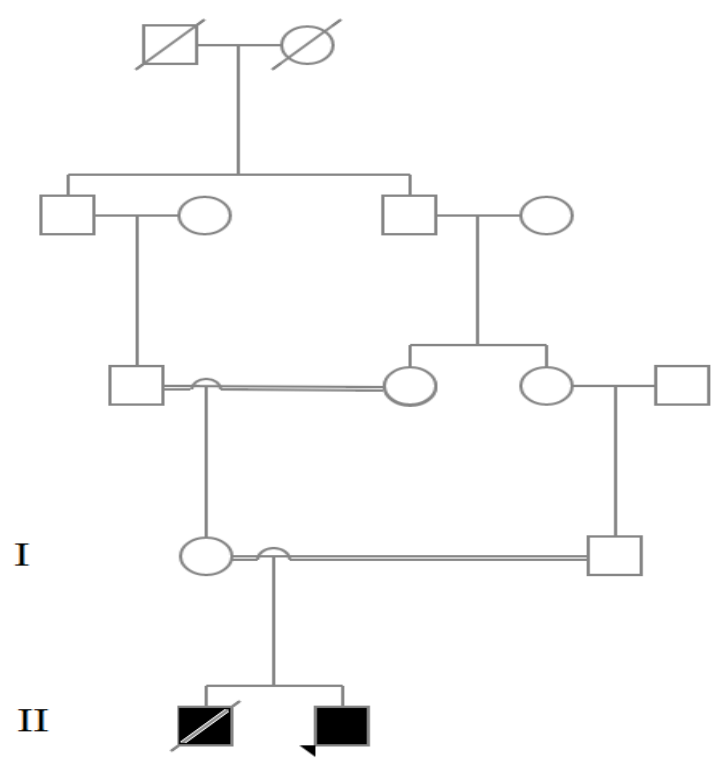

Figure 1. Family pedigree 


\section{Result}

\subsection{Clinical reports for the patient}

The enrolled family belonged to the Lor ethnicity of Iran. The proband in the family was a boy (Figure 1, II2) with 8 years of age. Parents (Figure 1, II-1, and II-2) were the first cousins. The first son of the family had the same symptoms as proband. The proband represented developmental delay and regression, sensory-neural hearing loss, myoclonic epilepsy, 3-methylglutaconic aciduria, mental retardation, respiratory problems, jaundice, dysphagia, and lesion in basal ganglia.
The metabolic test report for the proband was as follows: The organic acids in the patient's urine showed a moderate excretion of 3-methyglutaric acid (16 mmol/ mol creatinine, normally not detected) and 3-methylglutaconic acid $(79 \mathrm{mmol} / \mathrm{mol}$ creatinine, normal $<19)$.

The following magnetic resonance imaging results of the brain for the proband were obtained: increased signal intensity in the deep white matter of the brain in T2weighted images is more probably representative of the immaturity of the neural migration; however, the basal ganglia with increased signal in T1-weighted images possibly indicate the metabolic disease of the brain.

Table 2. Location and predicted pathogenicity of the variation. Data were extracted by the Mutation taster and DDIG-in

\begin{tabular}{|c|c|c|c|}
\hline Gene & Mutation & Mutation taster & DDIG-in \\
\hline SERAC1 & $\begin{array}{c}\text { chr6, } 158571484 \text { C > CCATG g.17828- } \\
\text { 17829insCATG }\end{array}$ & $\begin{array}{l}\text { Disease-causing } \\
\quad(\text { prob }>0.99)\end{array}$ & $\begin{array}{c}\text { Disease } \\
\text { (prob=86.51) }\end{array}$ \\
\hline
\end{tabular}

NEUR SCIENCE
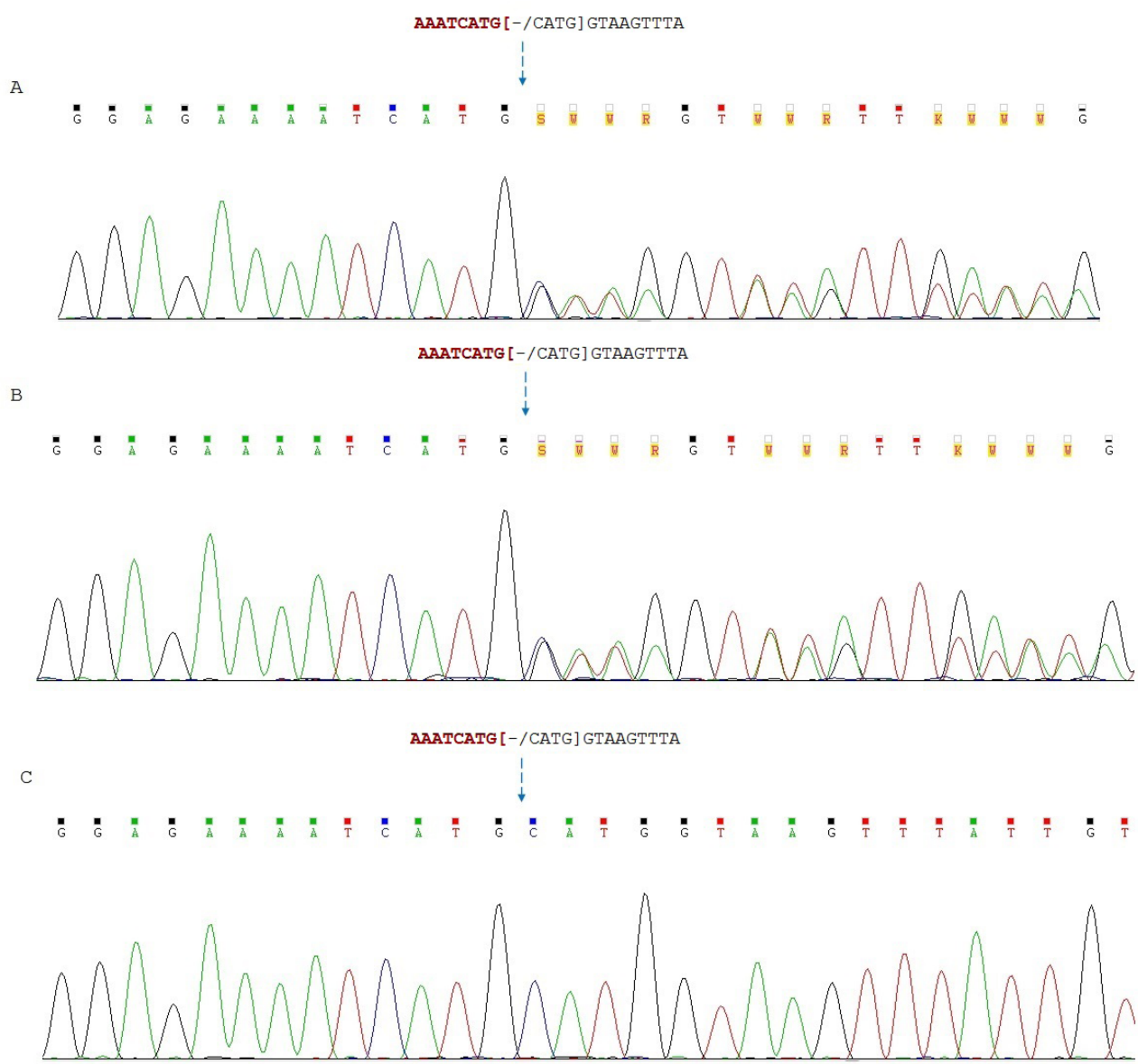

NEUR SCIENCE

Figure 2. Chromatograms of the affected child's and his parents' genome by focusing on the targeted region, including the mutation; A: Father; B: Mother; and C. Affected child 

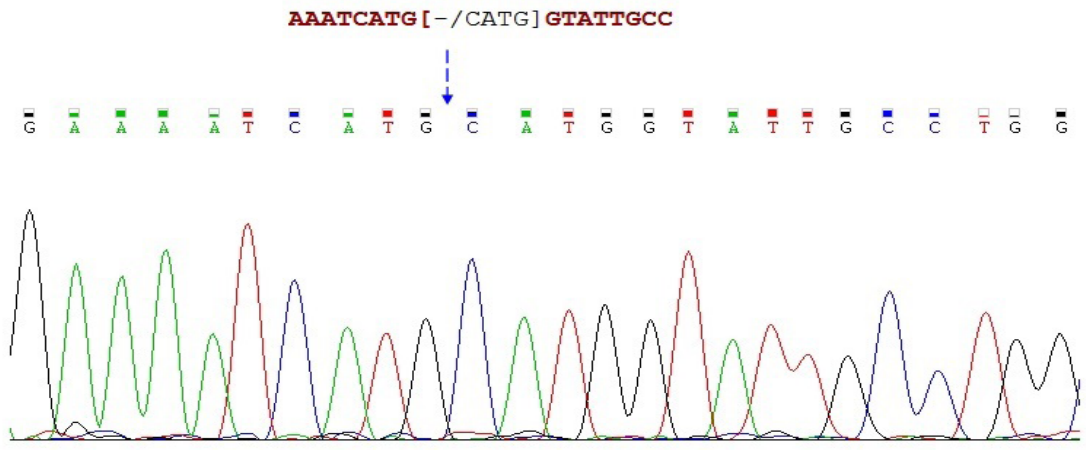

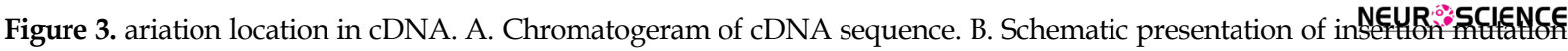
in DNA \& spliced mRNA/cDNA

\subsection{Variant characteristics}

Following WES data analysis, we identified g.1782817829insCATG homozygous single-nucleotide variation in the patient in the SERAC1 gene.

There are 25 reported variants in the ClinVar database (https://www.ncbi.nlm.nih.gov/clinvar/) for the SERAC1 gene, including 8 benign, 3 likely benign, 3 likely pathogenic, and 11 pathogenic variants (Table 3 ). The present variant, rs797045105 (Table 2, g.1782817829insCATG) reported in ClinVar as likely pathogenic variant provided by clinical testing.

Table 3. SERAC1 variants in ClinVar database

\begin{tabular}{|c|c|c|}
\hline Variant & Condition (s) & Clinical Significance \\
\hline 2-BP DUP, 1627TC & MEGDEL syndrome & Pathogenic \\
\hline 3-BP DEL, 1435CTT & MEGDEL syndrome & Pathogenic \\
\hline 4-BP DEL, 1167TCAG & MEGDEL syndrome & Pathogenic \\
\hline IVS13DS, G-C, +1 & MEGDEL syndrome & Pathogenic \\
\hline NM_032861.3:c.1916G>C (p.Arg639Pro) & Not provided & Pathogenic \\
\hline NM_032861.3c.1628_1629dupCT (p.Val544Leufs) & Not provided & Pathogenic \\
\hline NM_032861.3c.1501+3_1501+6delAAGT & Not provided & Likely pathogenic \\
\hline NM_032861.3:c.1347_1350dupATCT (p.Val451llefs) & Not provided & Pathogenic \\
\hline NM_032861.3:c.1126C>T (p.Gln376Ter) & Not provided & Pathogenic \\
\hline NM_032861.3:c.442C>T (p.Arg148Ter) & MEGDEL syndrome & Pathogenic \\
\hline NM_032861.3:c.265_265+1insCATG & MEGDEL syndrome & Likely pathogenic \\
\hline NM_032861.3:c.202C>T (p.Arg68Ter) & MEGDEL syndrome & Pathogenic \\
\hline NM_032861.3:c.21C>G (p.Cys7Trp) & Not provided & Likely pathogenic \\
\hline
\end{tabular}

SERAC1 protein (NP_116250) has 654 amino acids, including 32-54 amino acids in transmembrane regions, 386-592 as pimeloyl-ACP methyl ester carboxylesterases, and 396-540 as lipases/esterases. Up to now, most of the reported mutations occurred in the lipases/esterases.

3.3. Sanger sequencing analysis of genomic amplification

We proved the result of WES of the patient using Sanger sequencing. Also, allele segregation was checked by parents' genome amplification and sequence assessment of the targeted region included the variation. The results showed that the patient was homozygous and his parents were heterozygous for the present variant (Figure 2). 


\section{4. cDNA analysis}

We demonstrated the insertion of nucleotides in the transcripts of SERAC1 using real-time (RT)-PCR and Sanger sequencing of cDNA product of SERAC1 transcripts (Figure 3).

\subsection{ORF finder}

We used the Open Reading Frame (ORF) Finder to search potential protein-encoding segments of the wild type and mutant cDNA of SERAC1. The result of ORF Finder showed that the mutant sequence cannot produce correct protein-coding frames.

\section{Conclusion}

Neurometabolic disorders are complex because they are genetically heterogeneous, with different genetic defects that give rise to clinically indistinguishable phenotypes. Finding more pathogenic mutations is effective to identify the disease characteristics and make a progress in its diagnosis and treatment. WES is a useful approach in the diagnosis of such complex disorders (Kaname, Yanagi, \& Naritomi, 2014; Rabbani, Tekin, \& Mahdieh, 2014, Tarailo-Graovac, et al., 2016).

The 3-MGA-uria type IV, a heterogeneous group of inborn metabolic errors with primary mitochondrial and endoplasmic reticula impairment, leads to a spectrum of multisystem defects. Various enzymes, structural proteins, cellular transport proteins, and other constituents are responsible for neurometabolic disorders including 3-MGAuria (Wortmann, et al., 2013; Wortmann, et al., 2013).

Recently, SERAC1 mutations have been found to cause MEGDEL syndrome (3-MGA-uria type IV). The symptoms of the disease are central nervous system involvement, neurological deterioration, microcephaly, sensorineural hearing loss, encephalopathy, dystonia, optic atrophy, and abnormity in the range of urine 3-MGA, plasma lactate, alanine, and cholesterol (Wortmann, et al., 2012, Tort, et al., 2013). SERAC1 has a role in the biosynthetic process of phospholipids and is an important player in the mitochondria and endoplasmic reticulum functions.

In the present study, we carried out WES to investigate the causative genetic mutations in an Iranian patient suspicious of MEGDEL syndrome. Following data analysis, we identified a frameshift homozygous variation, g.17828-17829insCATG (reported before as rs797045105, in the patient. This variant was heterozy- gous in the parents. Also, the identified variant was not present in 700 exome files sequenced from 700 referred patients to our laboratory in the southwest of Iran.

In conclusion, rs797045105, which has been reported clinically in the ClinVar database as a likely pathogenic variant could be considered as a pathogenic variant causing MEGDEL syndrome. The variant was located is in the boundary of exon 4 and introns 4-5. Interestingly, Sanger sequencing of SERAC1 cDNA confirmed the insertion of nucleotides in the spliced transcripts of the gene; therefore, it is predicted to be pathogenic due to the production of p.Gly89fs. SERAC1 encodes a protein with a serine-lipase domain. This variation disrupts the potential of SREAC1 mRNAs for coding this domain.

Our results are in line with many studies conducted recently regarding the effectiveness of WES as an appropriate tool for the diagnostic of highly heterogeneous neurometabolic disorders.

\section{Ethical Considerations}

\section{Compliance with ethical guidelines}

This research study was approved by the Ahvaz Jundishapur University of Medical Sciences Institutional Review Boards. For WES studies, informed consent was obtained. All experiments were performed in accordance with relevant guidelines and regulations.

\section{Funding}

This research did not receive any specific grant from funding agencies in the public, commercial, or not-forprofit sectors.

\section{Authors' contributions}

Mina Zamani, Hamid Galehdari, Gholamreza Shariati and Alireza Sedghat conceived and designed the study. Mina Zamani drafted the manuscript. Mina Zamani, Tahereh Seifi, Jawaher Zeighami, Neda Mazaheri and Hamid Galehdari analyzed and interpreted the data. Mina Zamani, Tahereh Seifi, Jawaher Zeighami, Emad Jahangirnezhad, and Minoo Gholamzadeh acquired the data.

\section{Conflict of interest}

There is no conflict of interest regarding the publication of this article. 


\section{Acknowledgments}

We acknowledge patient and his parents for their cooperation and participation in the study. We appreciate all the members of Narges Genetic lab.

\section{References}

Kaname, T., Yanagi, K., \& Naritomi, K. (2014). A commentary on the promise of whole-exome sequencing in medical genetics. Journal of Human Genetics, 59(3), 117-8. https:/ /www nature.com/articles/jhg20147

Rabbani, B., Tekin, M., \& Mahdieh, N. (2014). The promise of whole-exome sequencing in medical genetics. Journal of $\mathrm{Hu}$ man Genetics, 59(1), 5-15. https:/ / www.nature.com/articles/ jhg2013114

Tarailo-Graovac, M., Shyr, C., Ross, C. J., Horvath, G. A., Salvarinova, R., \& Ye, X. C., et al. (2016). Exome sequencing and the management of neurometabolic disorders. New England Journal of Medicine, 374(23), 2246-55. https:/ / www.nejm.org/ doi/full/10.1056/NEJMoa1515792

Tort, F., García-Silva, M. T., Ferrer-Cortès, X., Navarro-Sastre, A., Garcia-Villoria, J., \& Coll, M. J., et al. (2013). Exome sequencing identifies a new mutation in SERAC1 in a patient with 3-methylglutaconic aciduria. Molecular Genetics and Metabolism, 110(1-2), 73-7. [DOI:10.1016/j.ymgme.2013.04.021]

Wortmann, S., Rodenburg, R. J. T., Huizing, M., Loupatty, F. J., De Koning, T., \& Kluijtmans, L. A. J., et al. (2006). Association of 3-methylglutaconic aciduria with sensori-neural deafness, encephalopathy, and Leigh-like syndrome (MEGDEL association) in four patients with a disorder of the oxidative phosphorylation. Molecular Genetics and Metabolism, 88(1), 47-52. [DOI:10.1016/j.ymgme.2006.01.013.]

Wortmann, S. B., Duran, M., Anikster, Y., Barth, P. G., Sperl, W. \& Zschocke, J., et al. (2013). Inborn errors of metabolism with 3-methylglutaconic aciduria as discriminative feature: proper classification and nomenclature. Journal of Inherited Metabolic Disease, 36(6), 923-8. [DOI:10.1007/s10545-012-9580-0.]

Wortmann, S. B., Kluijtmans, L. A., Engelke, U. F., Wevers, R. A., \& Morava, E. (2012). The 3-methylglutaconic acidurias: what's new? Journal of Inherited Metabolic Disease, 35(1), 13-22. [DOI:10.1007/s10545-010-9210-7]

Wortmann, S. B., Kluijtmans, L. A., Rodenburg, R. J., Sass, J. O., Nouws, J., \& Van Kaauwen, E. P., et al. (2013). 3-Methylglutaconic aciduria--lessons from 50 genes and 977 patients. Journal of Inherited Metabolic Disease, 36(6), 913-21. [DOI: 10.1007/ s10545-012-9579-6.]

Wortmann, S. B., Vaz, F. M., Gardeitchik, T., Vissers, L. E., Renkema, G. H., \& Schuurs-Hoeijmakers, J. H., et al. (2012). Mutations in the phospholipid remodeling gene SERAC1 impair mitochondrial function and intracellular cholesterol trafficking and cause dystonia and deafness. Nature Genetics, 44(7), 797-802. [DOI:10.1038/ng.2325.] 
This Page Intentionally Left Blank 